\title{
High-pressure behavior of Ni-filled and Fe-filled multiwalled carbon nanotubes
}

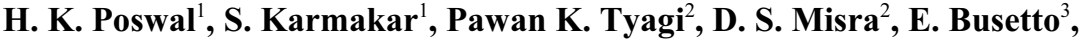 \\ Surinder M. Sharma ${ }^{*},{ }^{1}$, and A. K. Sood ${ }^{4}$ \\ 1 High Pressure Physics Division, Bhabha Atomic Research Centre, Mumbai 400085, India \\ 2 Department of Physics, Indian Institute of Technology, Powai, Mumbai 400076, India \\ 3 Syncrotron Trieste - Elettra, S.S.-14 Km 163,5 in Area Science Park, Basoviza, 34012 Trieste, Italy \\ ${ }^{4}$ Department of Physics, Indian Institute of Science, Bangalore 560012, India
}

Received 15 December 2006, revised 12 June 2007, accepted 20 June 2007

Published online 3 August 2007

PACS 61.10.Nz, 61.46.Fg, 62.25.+g, 62.50.+ p, 81.07.De

The high-pressure behavior of Ni-filled and Fe-filled multiwalled carbon nanotubes has been investigated up to $27 \mathrm{GPa}$ and $19 \mathrm{GPa}$, respectively, with the help of synchrotron-based angle-dispersive X-ray diffraction. These nanotubes do not show any structural transformation up to the highest pressures studied. These results are similar to that of Co-filled nanotubes [1], but are in sharp contrast to the earlier results on Fefilled tubes [2], where the tubes were found to undergo a sudden collapse of intertube separation. Our present observations suggest that the occurrence of an isostructural phase transition in $\mathrm{Fe}_{3} \mathrm{C}$, observed in our earlier study, may be responsible for the structural transition in nanotubes in that case.

(C) 2007 WILEY-VCH Verlag GmbH \& Co. KGaA, Weinheim

\section{Introduction}

Due to the possibilities of substantially different behavior compared to the bulk, the studies of nanocrystalline materials under high pressures are of considerable current interest. Nanocrystalline $\mathrm{CdSe}, \mathrm{Si}$, $\mathrm{Al}_{2} \mathrm{O}_{3}$ and $\mathrm{Fe}$ show pressure-induced structural phase transitions, some of which are different from the bulk [3-6]. Even when the transformations are same, the pressures at which structural changes occur can be quite different from that in the bulk [3-7]. For example, the diamond to beta-tin phase change in silicon nanorods occurs at much lower pressures than in bulk [7]. On the other hand, an increase in the transformation pressure has been observed in the case of spherical Si nanoparticles $[3,6]$.

There is another class of nanosystems that are quite interesting due to their unique features, i.e. nanotubes and nanowires. In particular, due to the high mechanical strength and ballistic electronic conduction, carbon nanotubes are beginning to find several uses such as for scanning probes [8], electronic transistors $[9,10]$, field-emitting devices and energy storage [11, 12]. Nanotubes can also be filled with biological molecules, raising the possibility of applications in biotechnology [13]. Encapsulation of various metals in multiwalled carbon nanotubes (MWCNTs) is being used to study the physical properties of nanowires and nanoparticles of these metals [14-16]. Transition-metal nanowires, encapsulated inside the multiwalled carbon nanotubes are promising materials for use in nanodevices, in the magnetic storage industry and for spintronics materials [17]. Several interesting results have been obtained on filled MWCNTs. For example, Fe-filled MWCNTs were shown to behave as a one-dimensional exchange-

* Corresponding author: e-mail: smsharma@barc.gov.in 
coupled ferromagnetic system [16]. Recently, Sun et al. [18] have shown that controlled irradiation of MWCNTs can cause large pressure buildup within the nanotube cores that can plastically deform, extrude and even break the solid materials encapsulated inside the core. With the help of atomistic simulations they also showed that the internal pressure inside nanotubes may be as large as $40 \mathrm{GPa}$. The electron-irradiation studies on multishell fullerene clusters (carbon onions) encapsulating iron nanocrystals demonstrated the formation of $\mathrm{Fe}_{3} \mathrm{C}$ (cementite) in the core of the cluster. They have argued that the formation of $\mathrm{Fe}_{3} \mathrm{C}$ in these spherical nanoencapsulates may be due to high pressure, mobile carbon interstitial atoms and the irradiation-induced lowering of kinetic barrier [19]. A useful review of high-pressure studies on nanomaterials, including nanotubes has been given by San-Miguel [20].

Our previous high pressure structural investigations on iron-filled carbon nanotubes showed several interesting results [2]. In particular, these studies showed that the filled nanotubes, unlike pristine tubes, show sudden collapse of the tubes' cross section (probable polygonization) at a pressure where the interfacial part of the nanowires, i.e. $\mathrm{Fe}_{3} \mathrm{C}$ undergoes an isostructural phase transition. Due to the coincident nature of these transformations, the driving mechanism remained unclear. In addition, the compressibilities of both the components of the nanowires $\left(\mathrm{Fe}\right.$ and $\left.\mathrm{Fe}_{3} \mathrm{C}\right)$ were found to be higher than the bulk counterparts. In contrast to this, our recent high-pressure structural studies on cobalt-filled multiwalled carbon nanotubes [1] did not show any phase transition in the MWCNTs up to the highest pressure ( $40 \mathrm{GPa}$ ). However, the cobalt nanowires encapsulated in the multiwalled carbon nanotubes showed a phase transition from fcc to hep phase at $\sim 9 \mathrm{GPa}$ [1]. In order to gain a further understanding of the high-pressure behavior of encapsulated metals and nanotubes, more studies of such systems would be useful. Recently, single-crystalline nickel and iron nanowires (having aspect ratios $\sim 100$ ), encapsulated in MWCNTs, have been synthesized by the MPCVD technique [21]. In comparison with the metal-filled tubes made through the pyrolysis method [2], the tubes made using MPCVD and used in the present investigation (as also are Co-filled nanotubes) do not have an interfacial carbide layer [21]. We present here the results of our high-pressure synchrotron-based angle-dispersive X-ray diffraction measurements on these $\mathrm{Ni}$ - and Fe-filled tubes.

\section{Experimental details}

Nickel-filled (Fe-filled) multiwalled carbon nanotubes were prepared from nickel (iron) nanoclusters, formed by the ammonia plasma treatment of the electroplated nickel (iron), using microwave plasma chemical vapor deposition (MPCVD) method. These nanowires were characterized using X-ray diffraction and high-resolution TEM (HRTEM). Ni-filled tubes were estimated to have a filling factor of $40-50 \%$, while Fe filled tubes were comparatively less filled (15-20\%). Encapsulated metallic nanowires are found to be single crystalline in nature, with crystallographic planes inclined at specific angles with respect to the tube axis so as to minimize elastic deformation energies [21]. Moreover, no interfacial carbide formation has been observed in these tubes. More details of the method of preparation and characterization are given in Ref. [21].

For the high-pressure experiments nickel-filled or Fe-filled MWCNTs were loaded along with a few particles of copper, in a hole of $\sim 120 \mu \mathrm{m}$ diameter drilled in a pre-indented $\sim 70 \mu \mathrm{m}$ tungsten gasket of a Mao-Bell-type diamond-anvil cell (DAC). Methanol: ethanol:water mixture $(16: 3: 1)$ was used as pressure-transmitting medium, which provides hydrostatic pressure environment up to $\sim 15 \mathrm{GPa}$ [22]. The pressure was determined from the known equation of state of copper [23]. High-pressure angledispersive X-ray-diffraction experiments were carried out at the 5.2R (XRD1) beamline of the Elettra synchrotron source with monochromatized X-rays $(\lambda=0.6888 \AA$ in the case of Ni-filled and $0.64927 \AA$ in the case of Fe-filled samples). The diffraction patterns were recorded using a MAR345 imaging plate detector kept at a distance of $\sim 20 \mathrm{~cm}$ from the sample. Two-dimensional imaging plate records were transformed to one-dimensional diffraction profiles by the radial integration of diffraction rings using the FIT2D software [24]. 


\section{Results and discussion}

Figure 1 shows the angle-dispersive X-ray diffraction patterns of nickel nanowires encapsulated in the multiwalled carbon nanotubes at a few representative pressures. The diffraction peak observed at $2 \theta=11.72^{\circ}$ (marked as MWCNTs) is the characteristic peak for the multiwalled carbon nanotubes representing the average intertube separation. Similarly, Fig. 2 shows the X-ray diffraction patterns of ironfilled MWCNTs at a few representative pressures. Here, at the lowest pressure, a diffraction peak due to MWCNTs is observed at $\sim 11.06^{\circ}$. As the intertube separation is known to decrease with increasing diameter [25], the asymmetric line shape of the diffraction peak of MWNCTs may be due to a distribution of intershell distances in the MWCNTs. The average $d_{0}$ was determined by fitting the asymmetric line shape with a sum of a Gaussian and a Lorentzian. At ambient pressures, the deduced value of the average intershell spacing $\left(d_{0}\right)$ is $3.37 \AA$, which is same as the earlier reported values for the pristine MWCNTs $\left(d_{0}=3.37 \AA\right)$ [25]. However, it is slightly smaller than the values reported earlier for ironfilled $(3.42 \AA)$ and cobalt-filled MWCNTs $(3.43 \AA)$ [1,2]. This may be due to the larger inner diameter of the multiwalled tubes in this study, as has been verified by TEM results [21]. The variation of the intertube spacing $d_{0}$ (for both Ni-filled and Fe-filled tubes) with pressure is shown in Fig. 3. This figure does not display any sudden change in the pressure variation of the intertube separation. This is similar to the results of the Co-filled tubes [1], but is quite unlike the results of our previous study on Fe-filled carbon nanotubes where intertube separation was found to decrease abruptly at $\sim 9$ GPa [2]. As mentioned above, the Fe-filled MWCNTs used in the present study have been synthesized by the MPCVD technique and do not have the interfacial $\mathrm{Fe}_{3} \mathrm{C}$ present in our earlier tubes. It is interesting to note that very recently high-pressure $\mathrm{X}$-ray magnetic circular dichroism (XMCD) studies on $\mathrm{Fe}_{3} \mathrm{C}$ nanoparticles

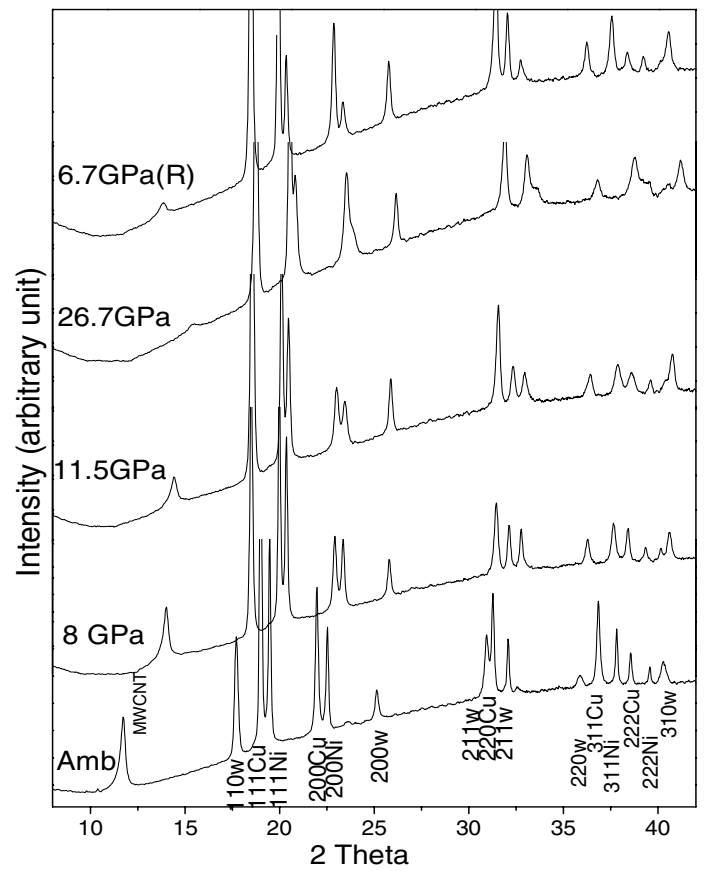

Fig. 1 X-ray diffraction patterns of Ni-filled MWCNTs at a few representative pressures. First peak denoted by MWCNT is the characteristic peak of multiwalled carbon nanotubes. $\mathrm{W}$ and $\mathrm{Cu}$ represent the diffraction peaks from the tungsten gasket and copper pressure calibrant, respectively.

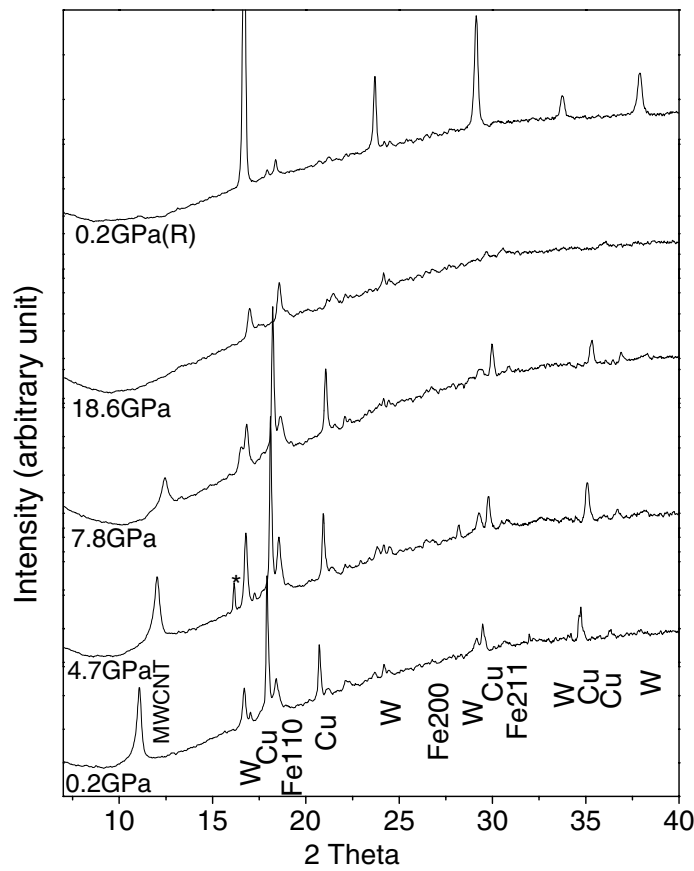

Fig. 2 X-ray diffraction patterns of Fe-filled MWCNTs at a few representative pressures. First peak denoted by MWCNT is the characteristic peak of multiwalled carbon nanotubes. $\mathrm{W}$ and $\mathrm{Cu}$ represent the diffraction peaks from the tungsten gasket and copper pressure calibrant, respectively. 


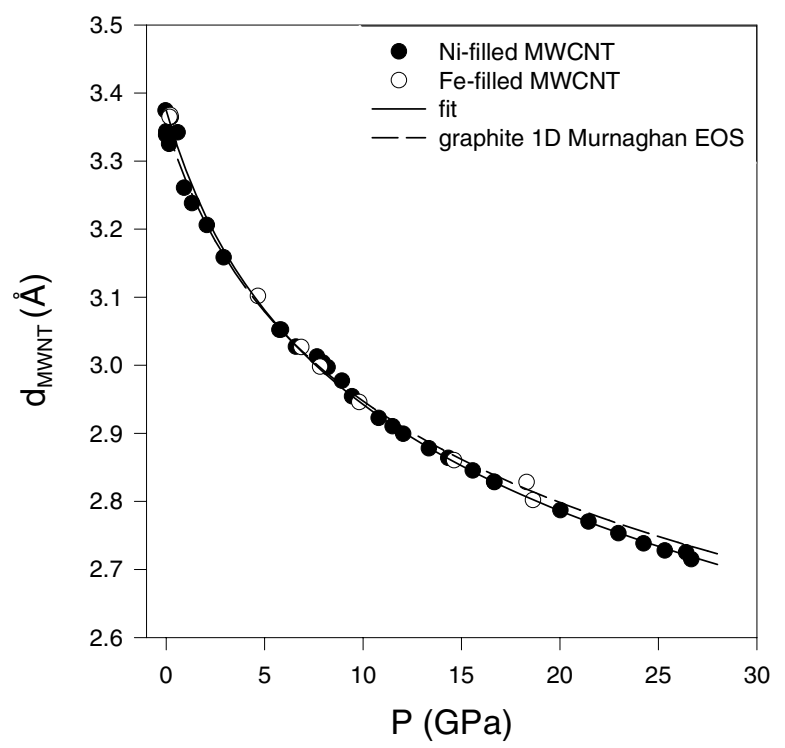

Fig. 3 Variation of intershell separation $\left(d_{0}\right)$ of MWCNTs (for Ni-filled and Fe-filled) with pressure. Solid line is a least square fit to equation [1] and the dotted line is the corresponding one dimensional equation of state of graphite (along the $c$-axis) [29].

have shown high moment to low moment transition at $\sim 10 \mathrm{GPa}$ [26]. Although so far no structural investigation is reported on unencapsulated nanocrystalline $\mathrm{Fe}_{3} \mathrm{C}$, our previous studies on Fe-filled nanotubes [2] showed that $\mathrm{Fe}_{3} \mathrm{C}$ in the nanowires undergoes an isostructural transition at $\sim 9 \mathrm{GPa}$. Therefore, the sudden collapse in the intertube separation in our previous study on Fe-filled MWCNT might have been triggered by the isostructural phase transformation of the nanocrystalline interfacial $\mathrm{Fe}_{3} \mathrm{C}$, which was also associated with a reduction in the compressibility.

From the width (FWHM) of the Bragg peak of MWCNTs and using Scherrer's formula, we estimate the total wall thickness of the MWNCT to be $\sim 10 \mathrm{~nm}$, which agrees well with the results of TEM studies [21]. Figure 4 shows that the peak width of Ni- and Fe-filled MWCNTs increases sharply beyond $9 \mathrm{GPa}$, a feature similar to what has been observed earlier in the pristine tubes $[2,27]$. As the width of the copper pressure calibrant (also given in Fig. 4) hardly changes up to $\sim 27 \mathrm{GPa}$, it is reasonable to assume that

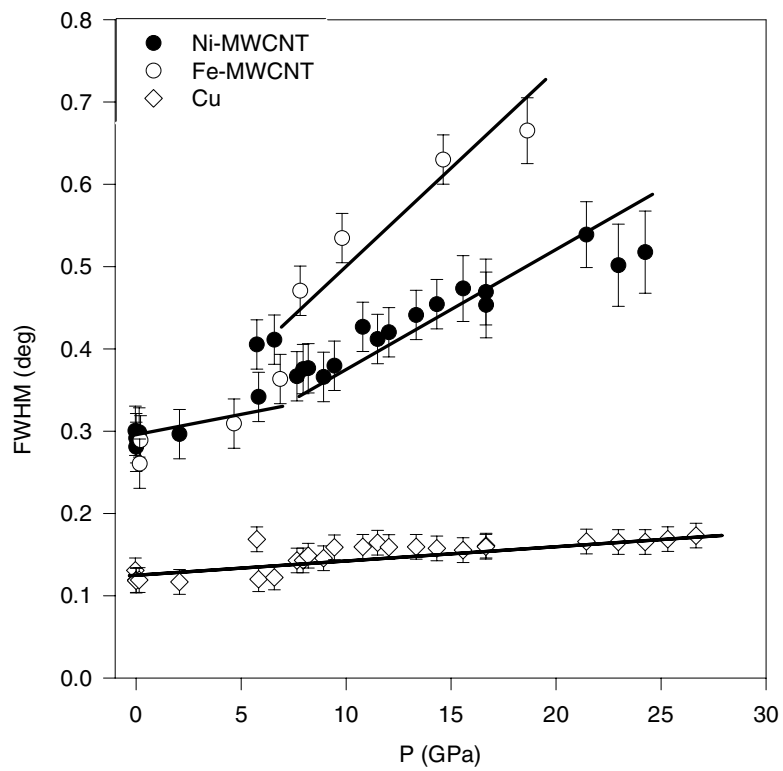

Fig. 4 Pressure-induced changes of the full width at half maxima of the diffraction peaks corresponding to MWCNTs and copper (pressure calibrant). 
Table 1 Comparison of $B_{0}$ and $B^{\prime}$ of encapsulating and pristine multiwalled carbon nanotubes with that of graphite.

\begin{tabular}{lll}
\hline & $B_{0}(\mathrm{GPa})$ & $B^{\prime}$ \\
\hline graphite & 35.7 & $10.8[29]$ \\
Ni-filled MWCNT & $33.1 \pm 5$ & $10.3 \pm 1.4$ [present study] \\
Fe-filled MWCNT & $35 \pm 2$ & 10.2 [present study] \\
$\begin{array}{l}\text { Fe-filled MWCNT } \\
\quad \text { having interfacial Fe }\end{array}$ C) & 49.3 & $8.1[2]$ \\
$\begin{array}{l}\text { Co-filled MWCNT } \\
\text { pristine MWCNT }\end{array}$ & $50 \pm 3$ & $5.5 \pm 0.7[1]$ \\
\hline
\end{tabular}

the observed sharp increase of the width of MWCNTs diffraction peak is not due to the nonhydrostatic stresses. Therefore, this change may be ascribed to the partial disorder or heterogeneous deformation of the tubes, as speculated earlier [27]. The higher slope of increase of FWHM of Fe filled tubes, compared to that of Ni-filled tubes, may be due to less filling of those tubes. This is supported by the observation that this is intermediate between those of Ni-filled and pristine MWCNTs [28].

The pressure-induced compression of nickel-filled and iron-filled MWCNTs when fitted to the onedimensional Murnaghan equation [29]

$$
\frac{a}{a_{0}}=\left[\left(\frac{B^{\prime}}{B_{0}}\right) P+1\right]^{\left(-\frac{1}{B^{\prime}}\right)},
$$

gives $B_{0}=33.1 \pm 5 \mathrm{GPa}, B^{\prime}=10.3 \pm 1.4$ and $B_{0}=35 \pm 2 \mathrm{GPa}, B^{\prime}=10.2$, respectively. These values differ significantly from the results of earlier iron-filled [2], cobalt-filled MWCNTs [1], and pristine MWCNTs for which $B_{0}=49.3 \mathrm{GPa}, B^{\prime}=8.1$ and $B_{0}=50 \pm 3 \mathrm{GPa}, B^{\prime}=5.5 \pm 0.7$ and $B_{0}=40.1 \pm 3 \mathrm{GPa}$ and $B^{\prime}=10.8$ [27], respectively. However, for Ni-filled MWCNTs, the results are very close to the values for the linear moduli along the $c$-axis of graphite $\left(B_{0}=35.7 \mathrm{GPa}\right.$ and $\left.B^{\prime}=10.8\right)$ [29]. This is understandable, as the diameter of the innermost tube in the present case is larger than the earlier cases, supporting a more graphite-like behavior. For easy comparison these values have been tabulated in Table 1. We should also mention here that as the intensity of MWCNT diffraction peaks reduces beyond $\sim 10 \mathrm{GPa}$, the errors in $d_{0}$ increase beyond this pressure.

The absence of either vanishing or appearance of any diffraction line in the diffraction pattern shown in Fig. 1, implies that the encapsulated Ni does not undergo any phase transition up to the highest pressure in this experiment, i.e. $27 \mathrm{GPa}$. The pressure-induced variation in the lattice parameter was determined by analyzing the diffraction patterns with the help of Le-Bail profile fitting [30]. In the case of Ni-filled tubes, multiphase analysis was performed incorporating three phases, viz., fcc-nickel, fcccopper and bcc-tungsten. At ambient conditions, the lattice parameter of nickel nanowires encapsulated

Table 2 Comparison of $B_{0}$ and $B^{\prime}$ of bulk, nanocrystalline and encapsulated Ni-nanowires.

\begin{tabular}{lll}
\hline & $B_{0}(\mathrm{GPa})$ & $B^{\prime}$ \\
\hline bulk Ni & $161 \pm 11$ & $7.5 \pm 1[4]$ \\
& 180 & 4 (fixed) [4] \\
nanocrystalline Ni & $177 \pm 2$ & 4 (fixed) [38] \\
& $185.4 \pm 10$ & 4 (fixed) [4] \\
encapsulated Ni nanowire & $161 \pm 3$ & 4 (fixed) [38] \\
& $179.8 \pm 24.7$ & $5.3 \pm 3.1$ (present study) \\
& $190.4 \pm 4$ & 4 (fixed) (present study) \\
\hline
\end{tabular}




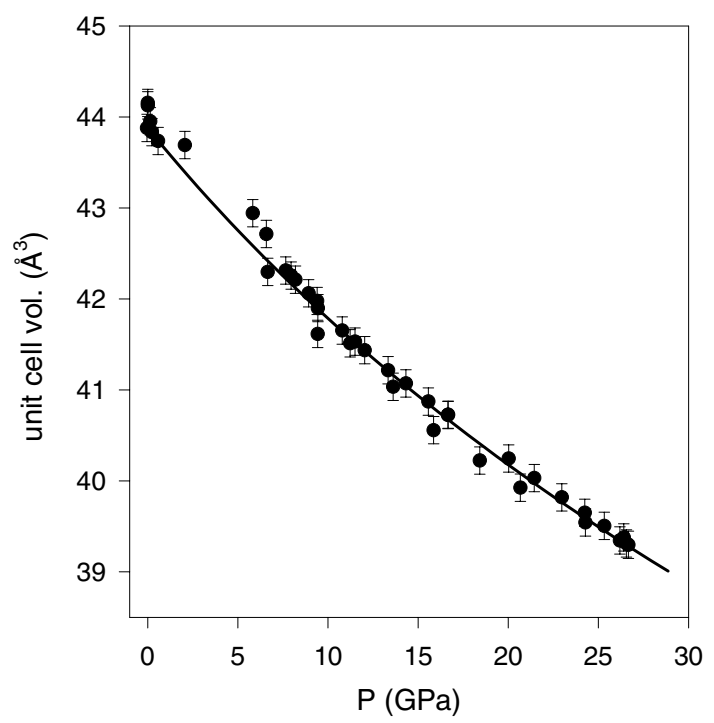

Fig. 5 Variation of unit-cell volume of encapsulated Ni with pressure. Solid line is the fit to the BirchMurnaghan (BM) equation of state.

in the MWCNTs is found to be $3.527 \AA$, compared to the value of $3.52 \AA$ in bulk. A slightly higher value of the lattice parameters is a common feature of all nanoparticles [31]. The variation of the unit-cell volume with pressure is shown in Fig. 5. The $P-V$ behavior of encapsulated nickel when fitted to the Birch-Murnaghan equation of state [32], gives $B_{0}=179.8 \pm 24.7 \mathrm{GPa}$ and $B^{\prime}=5.3 \pm 3.1$. However, the bulk modulus obtained by constraining the $B^{\prime}$ to 4 , yields $B_{0}=190.4 \pm 4$. These results are consistent with those of an earlier high-pressure (energy dispersive) X-ray diffraction study on nanocrystalline nickel [4], where no phase transition was observed up to $55 \mathrm{GPa}$ (the highest pressure in that experiment) and the bulk modulus was found to be $B_{0}=185.4 \pm 10 \mathrm{GPa}$ with $B^{\prime}$ constrained to be 4 . For the bulk nickel, the bulk modulus is known to be $=161 \pm 11 \mathrm{GPa}$ with a $B^{\prime}=7.5 \pm 1$ [4]. And if $B^{\prime}$ is constrained to 4 , the fitted bulk modulus was found to be $180 \mathrm{GPa}$ [4]. So the compressibility of nanostructured nickel encapsulated within nanotubes is found to be comparable to that of the bulk. Also, the highpressure behavior of nickel nanowires is found to be similar to the behavior of nanocrystalline Ni studied earlier (particle size of $20 \mathrm{~nm}$ ). Table 2 gives the comparison between $B_{0}$ and $B^{\prime}$ of nickel nanowires, nanocrystalline nickel and the bulk.

In the case of Fe-filled MWCNTs, each diffraction pattern consists of the Bragg peaks due to MWCNTs (at $\sim 11.06^{\circ}$ ), bcc-iron, fcc-Cu and bcc-W. An additional feature (a few diffraction spots at $d$-spacing $=2.31 \AA$, shown by the asterisk $(*)$ in the diffraction pattern of Fig. 2) was found to appear at $\sim 5 \mathrm{GPa}$. With increasing pressure this evolved into a full diffraction ring that merged with the tungsten gasket peak at $\sim 10 \mathrm{GPa}$. The source of this behavior remains unclear at the moment. We have tried to fit the diffraction pattern at $\sim 5 \mathrm{GPa}$ with the known structures of iron (viz., hcp, fcc, orthorhombic and dhcp) or that of iron compounds such as $\mathrm{Fe}_{2} \mathrm{O}_{3}$ or $\mathrm{Fe}_{3} \mathrm{C}$, etc. However, none of these explains the emergence of this new diffraction peak. As mentioned earlier, it is interesting to note that this kind

Table 3 Comparison of $B_{0}$ and $B^{\prime}$ of bulk, and encapsulated Fe nanowires (for nanocrystalline $\mathrm{Fe}$, the bulk modulus for $\alpha$-phase has not been reported so far).

\begin{tabular}{lcc}
\hline & $B(\mathrm{GPa})$ & $B^{\prime}$ \\
\hline bulk Fe & 162.5 & $5.5[37]$ \\
encapsulated Fe nanowire & 89.7 & $20[2]$ \\
$\quad\left(\right.$ with interfacial $\left.\mathrm{Fe}_{3} \mathrm{C}\right)$ & & 8.5 (present study) \\
encapsulate Fe nanowire & $167 \mathrm{GPa}$ & \\
\hline
\end{tabular}




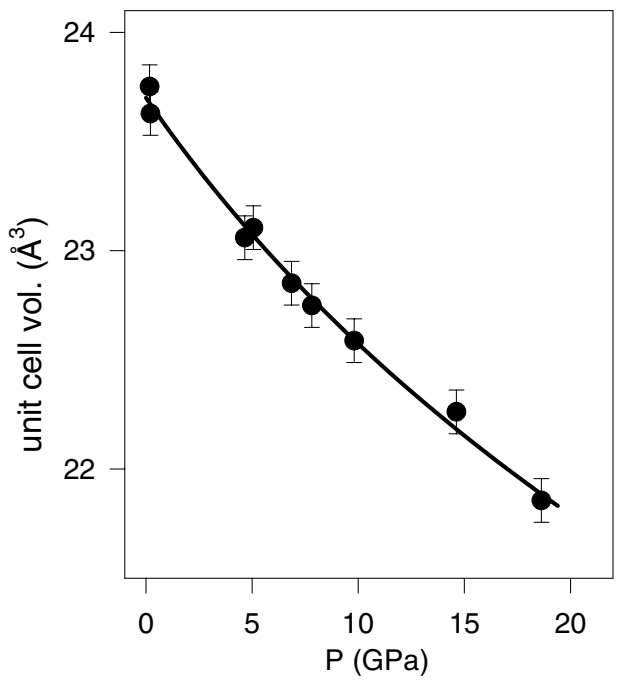

Fig. 6 Variation of unit-cell volume of encapsulated iron as a function of pressure. Solid line is the fit to the BirchMurnaghan (BM) equation of state.

of new feature was not observed in Ni-filled MWCNTs. The lattice constant of the bcc iron is found to be $\sim 2.875 \AA$. Like our previous studies [2], encapsulated Fe in this study also does not undergo any structural transition up to the highest pressure of this study $(\sim 19 \mathrm{GPa})$. In contrast, bulk and bare nanocrystalline iron undergoes a bcc-hep transition in the pressure range of $10-14 \mathrm{GPa}[33,34]$. One may speculate that this displacive phase transition may be suppressed because of the one-to-one matching of the carbon atoms of nanotubes with crystallographic planes of bcc $\mathrm{Fe}$, as in $\mathrm{Co}$ and $\mathrm{Ni}$ [35]. As the bcc to hcp transformation requires compression along the [001] and shearing of (110) layers along [1 $\overline{10}$ ] direction in the bcc phase [36], this transformation is likely to increase the elastic energy of the coupled system of nanotubes and Fe. Figure 6 shows pressure versus unit-cell volume for the bcc iron, which, when fitted to the Birch-Murnaghan equation of state, gives $B_{0}=167 \mathrm{GPa}$ and $B^{\prime}=8.5$, which shows that the bulk modulus of encapsulated bec-iron is similar to that of bulk iron (Table 3) [37].

To summarize, both Ni-filled and Fe-filled multiwalled carbon nanotubes do not undergo any first-order pressure-induced structural transformation in the pressure range of present investigations. The pressure-induced variation in the intertube separation is found to be comparable to that of graphite. Encapsulated Ni does not undergo any structural transition up to $27 \mathrm{GPa}$. Even the encapsulated Fe does not undergo the expected bcc-hcp structural transitions up to $19 \mathrm{GPa}$. The observed bulk moduli for Ni and Fe are found to be comparable to that of the bulk counterpart.

Acknowledgements The authors are thankful to P. Maurizio for his support during the experiments at XRD1 beamline of Elettra synchrotron. We also thank the Department of Science and Technology, Government of India, for financial support to carry out these experiments.

\section{References}

[1] S. Karmakar, P. K. Tyagi, D. S. Mishra, and S. M. Sharma, Phys. Rev. B 73, 184119 (2006).

[2] S. Karmakar, Surinder M. Sharma, P. V. Teredesai, and A. K. Sood, Phys. Rev. B 69, 165414 (2004).

[3] S. H. Tolbert, A. B. Herhold, L. E. Brus, and A. P. Alivisatos, Phys. Rev. Lett. 76, 4384 (1996).

[4] B. Chen, D. Penwell, and M. B. Kruger, Solid State Commun. 115, 191 (2000) and references therein.

[5] J. N. Wickham, A. B. Herhold, and A. P. Alivisatos, Phys. Rev. Lett. 84, 923 (2000).

[6] J. N. Wickham, A. B. Herhold, and A. P. Alivisatos, Phys. Rev. Lett. 84, 923 (2000).

[7] H. K. Poswal, N. Garg, S. M. Sharma, E. Busetto, S. K. Sikka, G. Gundiah, F. L. Deepak, and C. N. R. Rao, J. Nanosci. Nanotechnol. 5, 723 (2005).

[8] H. Dai, J. H. Hafner, A. G. Rinzler, D. T. Colbert, and R. E. Smalley, Nature 384, 147 (1996). 
[9] S. J. Trans, R. M. Veschueren, and C. Dekker, Nature 393, 49 (1998).

[10] P. G. Collins, A. Zettle, H. Bendo, A. Thess, and R. E. Smalley, Science 278, 100 (1997).

[11] W. A. de Heer, A. Chatelain, and D. Ugarte, Science 270, 1179 (1995).

[12] S. Fan, M. G. Chapline, N. R. Franklin, T. Tombler, A. M. Cassell, and H. Dai, Science 283, 512 (1999).

[13] M. Shim, N. W. S. Kham, R. J. Chen, and H. D. Y. Li, Nano Lett. 2, 285 (2002).

[14] M. A. Burns et al., Science 282, 484 (1998).

[15] C. N. R. Rao, B. C. Satishkumar, A. Govindaraj, and M. Nath, Chem. Phys. Chem. 2, 78 (2001).

[16] S. Karmakar, M. D. Mukadam, S. M. Yusuf, Surinder M. Sharma, and A. K. Sood, J. Appl. Phys. 97, 054306 (2005).

[17] Y. J. Kang, J. Choi, C. Y. Moon, and K. J. Chang, Phys. Rev. B 71, 115441 (2005).

[18] L. Sun, F. Banhart, A. V. Krasheninnikov, J. A. Rodriguez Manzo, M. Terrones, and P. M. Ajayan, Science 312, 1199 (2006).

[19] L. Sun and F. Banhart, Appl. Phys. Lett. 88, 193121 (2006).

[20] A. San-Miguel, Chem. Soc. Rev. 35, 876 (2006).

[21] P. K. Tyagi, A. Misra, M. K. Singh, D. S. Misra, J. Ghatak, P. V. Satyam, and F. Le Normand, Appl. Phys. Lett. 86, 253110 (2005).

[22] R. J. Angel, B. Maciej, Z. Jing, G. Diego, and S. D. Jacobson, J. Appl. Crystallogr. 40, 26 (2007) and references therein.

[23] A. Dewaele, P. Loubeyre, and M. Mezouar, Phys. Rev. B 70, 94112 (2004).

[24] A. P. Hammersley, S. O. Svensson, M. Hanfland, A. N. Fitch, and D. Hauserman, High Press. Res. 14, 235 (1996).

[25] C. H. Kiang, M. Endo, P. M. Ajayan, G. Dresselhaus, and M. S. Dresselhaus, Phys. Rev. Lett. 81, 1869 (1998)

[26] E. Duman, M. Acet, E. F. Wassermann, J. P. Itie, F. Baudelet, O. Mathon, and S. Pascarelli, Phys. Rev. Lett. 94, 075502 (2005).

[27] C. Liang-Chang, W. Li-Jun, T. Dong-Sheng, X. Si-Shen, and J. Chang-Qing, Chin. Phys. Lett. 18, 577 (2001).

[28] S. Karmakar, Ph.D. thesis, University of Mumbai (2006).

[29] M. Hanfland, H. Beister, and K. Syassen, Phys. Rev. B 39, 12598 (1989).

[30] A. C. Larson and R. B. Von Dreele, General Structure Analysis System (GSAS), Los Alamos National Laboratory Report LAUR 86, 748 (2004).

[31] B. Palosz, S. Gierlotka, S. Stel'makh, R. Pielaszele, P. Zinn, M. Winzenick, U. Bismayer, and H. Boysen, J. Alloys Compd. 286, 184 (1999).

[32] F. D. Murnaghan, Proc. Natl. Acad. Sci. USA 30, 244 (1944).

[33] R. Boehler, N. Vonbargen, and A. Chopelas, J. Geophys. Res. B 95, 21731 (1990).

[34] B. Chen, D. Penwell, M. B. Kruger, A. F. Yue, and B. Fultz, J. Appl. Phys. 89, 4794 (2001).

[35] In Ref. [21], it has been shown that to minimize elastic energy and the surface tension, the Miller planes of single-crystalline nickel and cobalt inside a carbon nanotube are inclined at specific angles with respect to the tube axis of the multiwall carbon nanotubes. At these angles, the corresponding interplanar spacing matches with the lattice of the carbon nanotubes. For example, the (111) planes of fcc nickel are inclined at angles of $39.6^{\circ}$ for which these planes are matched with the carbon atoms of graphene sheet.

[36] W. A. Basset and E. Huang, Science 238, 780 (1987).

[37] T. Takahashi, W. A. Bassett, and H. K. Mao, J. Geophys. Res. 73, 4717 (1968).

[38] Jianzhong Zhang and Yusheng Zhao, Appl. Phys. Lett. 90, 0443112 (2007). 